\title{
Exercise training reduces alcohol consumption but does not affect HPA-axis activity in heavy drinkers
}

\author{
Kalliopi Georgakouli ${ }^{\mathrm{a}, \mathrm{b}}$, Eirini Manthou ${ }^{\mathrm{a}, \mathrm{b}}$, Panagiotis Georgoulias ${ }^{\mathrm{c}}$, Anastasia Ziaka ${ }^{\mathrm{c}}$, \\ Ioannis G. Fatouros ${ }^{\mathrm{a}, \mathrm{b}}$, Georgios Mastorakos ${ }^{\mathrm{d}}$, Yiannis Koutedakis ${ }^{\mathrm{a}, \mathrm{b}}$, Yannis Theodorakis ${ }^{\mathrm{a}}$, \\ Athanasios Z. Jamurtas ${ }^{\mathrm{a}, \mathrm{b}, *}$ \\ a Department of Physical Education and Sport Science, University of Thessaly, Karies, Trikala 42100, Greece \\ b Institute of Human Performance and Rehabilitation, Centre for Research and Technology - Thessaly (CERETETH), Karies, Trikala 42100, Greece \\ ${ }^{\mathrm{c}}$ Department of Nuclear Medicine, Faculty of Medicine, University of Thessaly, 3 Panepistimiou, Viopolis, Larissa 41500, Greece \\ d Unit of Endocrinology, Diabetes Mellitus and Metabolism, Aretaieio Hospital, Athens Medical School, Athens University, 76 Vas. Sofias Avenue, Athens 11528, Greece
}

\section{A R T I C L E I N F O}

\section{Keywords:}

$\beta$-endorphin

Opioid system

Alcohol cessation

Fitness

Alcohol use disorders

HPA-axis

\begin{abstract}
A B S T R A C T
Background: It has been suggested that physical exercise could have potential beneficial effects in substance abusers, which are based on both physiological and psychological theories. Although a few studies have examined the effect of exercise on alcohol intake and fitness in individuals with alcohol use disorders (AUDs), there is a gap in the literature concerning the physiological and biochemical mechanisms that could be affected by physical exercise in this population.

Purpose: The purpose of the present study was to examine physiological and biochemical responses to exercise after an 8-week supervised exercise training (ET) intervention in heavy drinkers. The investigation was mainly focused on the relationship among exercise, opioids, the hypothalamic-pituitary-adrenal axis (HPA) activity and heavy alcohol drinking.

Methods: Eleven (Age: $30.3 \pm 3.5$ yrs; Body Mass Index: $28.4 \pm 0.86 \mathrm{~kg} / \mathrm{m}^{2}$ ) male heavy drinkers volunteered to participated in an 8-week supervised intervention of moderate intensity exercise (50-60\% of Heart Rate Reserve). All participants were exhibiting low physical activity and used to drink heavily. Before intervention, the participants were asked to record their daily alcohol intake without changing their physical activity levels for 4 weeks (control condition). During the 8-week supervised ET intervention, participants were recording their daily alcohol intake and were motivated to increase gradually the duration and frequency of ET. Blood samples were collected prior to and after 4 weeks of the control condition, the day before the beginning of the ET intervention, and at the end of the 4th and 8th week of ET intervention. Blood samples were analyzed for $\beta$-E, epinephrine, norepinephrine, adrenocorticotropin, cortisol, gamma-glutamyl transferase ( $\gamma$-GT), aspartate transaminase and alanine transaminase. Physiological and alcohol-related indices were also examined.

Results: The 8-week supervised ET intervention resulted in reduced alcohol consumption, reduced $\gamma$-GT levels, and fitness improvement in heavy drinker. ET intervention did not significantly change the hormonal responses. Conclusion: The results indicate that physical exercise could act as a healthy habit that can help individuals with AUDs reduce alcohol intake and improve health status; however, this is not related with changes in hormones associated with the HPA-axis.
\end{abstract}

\section{Introduction}

Light to moderate alcohol use is thought to have a beneficial impact on health (e.g., [1]), whereas uncontrolled and excessive alcohol consumption can negatively affect mental and physical health, and social aspects of humans [2]. Alcoholism and other disorders related to alcohol use are a major health concern, with alcohol abuse accounting for about $4.5 \%$ of all diseases and injuries worldwide [3].

Alcohol use has been reported to influence the activity of the endogenous opioid system of humans [4,5]. Acute exposure to alcohol may result in a fast and transient release of $\beta$-endorphin ( $\beta$-E) by the pituitary and hypothalamus [6,7] in a dose-dependent manner [8].

\footnotetext{
Abbreviations: AUD, Alcohol Use Disorder; AUDIT, Alcohol Use Disorders Identification Test; ET, Exercise Training

* Corresponding author at: School of Physical Education and Sport Science, University of Thessaly, Karyes, 42100 Trikala, Greece.

E-mail address: ajamurt@pe.uth.gr (A.Z. Jamurtas).
} 
Increased $\beta$-E levels, in turn, activate $\mu$ and $\delta$ receptors, possibly playing an important role in the reinforcing properties of alcohol intake. On the other hand, there may be a decreased $\beta$-E release as well as lower density and activity of the opioid receptors after chronic exposure to alcohol that may be responsible for feelings of discomfort and negative reinforcement $[4,5]$. This is based on reports that chronic alcohol abuse resulted in low concentration of $\beta$-E in the cerebrospinal fluid and plasma of alcoholics $[8,9]$. The endogenous opioid system is involved in the modulation of the HPA-axis. Since chronic alcohol abuse influences the activity of the endogenous opioid system, it may also be associated with impaired activity of the HPA axis and consequently increased levels of glucocorticoids. Indeed, excessive alcohol drinking is one of various stressful conditions that result in increased release of cortisol from the adrenal cortex and may be negatively associated with cognitive function [10]. Consequently, changes in HPA-axis activity may influence changes in the behavior towards alcohol use in individuals with alcohol use disorders (AUDs).

The low success rates of alcohol abuse treatment programmes and the high relapse rates are problems of great significance [11]. Substance abuse treatment includes changing deeply imbedded behaviors, involving lifestyle modifications. One lifestyle modification that has been proposed as an adjunct method for alcohol abuse cessation and prevention of relapse in alcoholics is physical exercise [11-14]. This statement is supported by research data indicating that pleasure ratings after exercise are higher compared to drinking alcohol in alcohol dependent patients [15]. Physical exercise is beneficial in many different ways; it enhances mood and psychological wellbeing [16], improves health and wellness, can be cost-effective, flexible, accessible, and have minimal side effects compared to pharmacological treatment [17].

There is limited research on the use of exercise as an adjunctive strategy in treatment programmes of AUDs. Available data from human studies have provided inconsistent results regarding alcohol abuse cessation, whereas fitness gains are more evident. The present study attempts to elucidate physiological responses to chronic exercise in heavy drinkers in order to understand the mechanisms that could potentially make physical exercise a promising tool in the treatment of AUDs.

\section{Material and methods}

\subsection{Subjects}

Subjects who participated in this study were recruited through flyers, posters, newspaper press releases, and word-of-mouth recruiting all over the region of Thessaly, Greece. To avoid confusion related to physiological differences between genders, all participants were men.

Exclusion criteria included: serious health conditions or physical disabilities or any other medical condition compromising safe participation in exercise; any person with a history of drug abuse other than alcohol; any person aged sixty and over.

All subjects were informed about the study protocol, the associated risks and benefits, and signed an informed consent form. They were all exhibiting low physical activity, as assessed by the Greek version of the International Physical Activity Questionnaire (IPAQ-Gr) [18], and they were identified as heavy alcohol drinkers by fulfilling at least one of the following criteria: (1) Drinking $>14$ drinks/week or 4 drinks per occasion; (2) Drinking 5 or more drinks on the same occasion on each of 5 or more days in the past 30 days; with 1 drink containing $14 \mathrm{~g}$ of pure alcohol [19]. Moreover, subjects completed the Alcohol Use Disorders Identification Test (AUDIT, [20]), which is a tool for identifying individuals with hazardous and harmful patterns of alcohol consumption [21]. AUDIT consists of 10 questions (scored individually from $0=$ never to $4=$ four or more times per week) about recent alcohol use, alcohol dependence symptoms, and alcohol-related problems [21]. Scores from 8 to 15 indicate hazardous drinking, which is a pattern of alcohol consumption that increases the risk of harmful consequences for the user or others [22]. Scores from 16 to 19 suggest harmful drinking, which is a pattern of alcohol consumption that leads to consequences to physical and mental health and maybe social consequences [22,23]. Scores of 20 or above indicate alcohol dependence [21].

After screening, 13 subjects fulfilled the criteria for participation. During the protocol intervention two of them dropped-out; one subject left the programme after completing the first 3 weeks of the protocol intervention due to personal reasons and another one was excluded from the study following the first five weeks of the protocol intervention due to low compliance with the programme. These subjects were identified as heavy drinkers according to the AUDIT and at baseline 4, 3 and 4 had a score of $8-15,16-19$, and 20 or above, respectively $(17.45 \pm 1.60$; mean $\pm \mathrm{SD}) .73 \%$ of them were smokers, while no history of other substance abuse was reported. In regards to employment, $27 \%$ of the subjects had been employed as professionals or skilled workers, $27 \%$ had been employed as unskilled workers, and $46 \%$ were university students at the time of enrolment to the study. Concerning personal life, $27 \%$ of the subjects were married, $9 \%$ were living with a partner, $18 \%$ were living with their parents and/or siblings, and $46 \%$ were living alone being single. Almost three quarters (73\%) of the subjects had secondary education according to the United Nations Educational, Scientific and Cultural Organization [24].

Moreover, baseline measurements were also conducted in a control group (CG), in order to set the normal values of $\beta$-E. The CG consisted of 11 matched controls (age: $34.1 \pm 2.0$ yrs) who were exhibiting low physical activity as well, but did not exceed the limits of moderate alcohol use and had a score of $<8$ at the AUDIT. Heavy drinkers and controls were also matched for smoking status; eight subjects in each group were current smokers.

\subsection{Experimental design}

Medical history was reviewed and a resting electrocardiogram (ECG) was performed on each subject in order to detect any heart abnormalities and contraindications to exercise. The procedures were in accordance with the 1975 Declaration of Helsinki and ethics approval was received from the University of Thessaly review board. This trial was registered at ClinicalTrials.gov as NCT02664766[25].

Before intervention, during a control condition period of 4 weeks all subjects were asked to record their daily alcohol intake while receiving no treatment. Subsequently an 8-week supervised exercise training (ET) intervention was carried out. During this intervention period, all subjects were also asked to record their daily alcohol intake and were motivated to gradually increase the duration and frequency of ET. All measurements of anthropometric, physiological, biochemical and alcohol-related parameters were performed prior to and after the control condition (pre/post) and prior to, after 4 and 8 weeks of the ET intervention (pre/mid/post) (Fig. 1).

\subsection{Anthropometric and physiological measurements}

Body weight was measured to the nearest $0.1 \mathrm{~kg}$ (Tanita Body Fat Monitor/Scale TBF-521; Tanita, Inc., IL, USA), with subjects lightly dressed and barefoot. Standing height was measured to the nearest $0.1 \mathrm{~cm}$ (Stadiometer 208; Seca, Birmingham, UK). Percentage body fat was assessed using the bioelectrical impedance analysis technique (Tanita Body Fat Monitor/Scale TBF-521; Tanita, Inc., IL, USA). Blood pressure (BP) was measured with a manual sphygmomanometer (FC101 Aneroid Sphygmomanometer; Focal Corporation, Japan). Heart rate was monitored by short-range telemetry (Polar RC3 GPS HR; Polar Electro Oy, Kempele, Finland) and was recorded every 5 min during exercise sessions. Body Mass Index (BMI) was calculated by the equation: $\mathrm{BMI}=\left(\right.$ weight in $\mathrm{kg}$ ) $/(\text { height in } \mathrm{m})^{2}$. Waist to hip ratio (WHR) was calculated by the equation: WHR = (waist circumference in $\mathrm{cm}$ ) / (hip circumference in $\mathrm{cm}$ ). Maximal oxygen uptake $\left(\mathrm{VO}_{2 \max }\right)$ was estimated based on a Single Stage Submaximal Treadmill Walking Test 


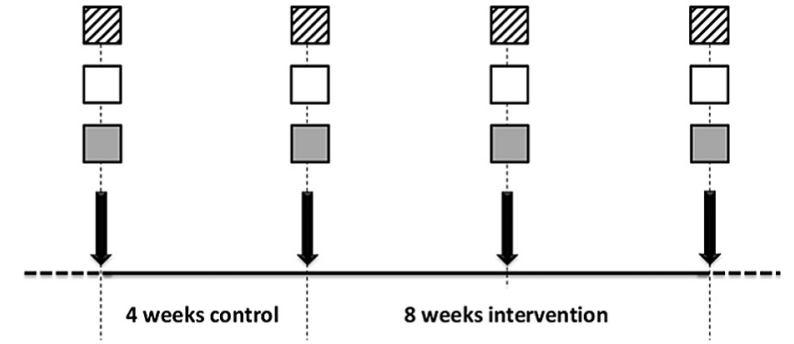

Blood sample collection

Physiological Assessments: Anthropometric Characteristics, Single Stage Submaximal Treadmill Walking Test, Sit \& Reach Test, Push-ups, Sit-ups, Hand Grip Test

Alcohol-related Assessments, Questionnaires for Alcohol Intake \& Urge, AUDIT, IPAQ

Fig. 1. Schematic presentation of the study protocol. AUDIT: alcohol use identification test; IPAQ: international physical activity questionnaire; $\beta$-E: beta endorphin; ACTH: adrenocorticotropic hormone; E: epinephrine; NE: norepinephrine, $\gamma$-GT: gamma-glutamyl transferase; AST: aspartate transaminase; ALT: alanine transaminase.

(SSTWT; [26]). Flexibility of the lower back and hamstrings was assessed using a sit and reach box [27]. Number of sit-ups performed in one minute, and number of push-ups until exhaustion were also recorded. Finally, the Hand Grip Test was performed with a digital handgrip dynamometer (T.K.K. 5710, Grip-D Takei, Tokyo, Japan) in order to assess handgrip strength.

\subsection{Blood collection and handling}

Participants were instructed to follow their usual eating habits and avoid any strenuous physical activity for at least two days before each blood sample collection. Participants were also asked to record their diet for two days before the first blood collection and follow the same diet before each of the subsequent blood samplings. All blood samples were collected in the morning (8:00-10:00 a.m.) after an overnight fast and smoking abstinence.

Blood samples were drawn from a forearm vein and then were handled as follows: Plasma and serum collection has been described elsewhere [28]. Moreover, a portion of blood was collected in vacutainer tubes containing EDTA and Trasylol ${ }^{\circledR}$ (aprotinin), shaken thoroughly and cooled in an ice-bath immediately until centrifugation $\left(1370 \times g\right.$ for $10 \mathrm{~min}$ at $\left.4{ }^{\circ} \mathrm{C}\right)$. The supernatant was transferred into Eppendorf tubes ${ }^{\circledast}$ and was immediately stored at $-80^{\circ} \mathrm{C}$ for later determination of $\beta$-E.

\subsection{Assays}

Analyses of all biologic substances were done in duplicates on the same day, while samples had undergone only one freeze-thaw cycle. Plasma catecholamines and $\beta$-E were determined by 125I-radioimmunoassay with commercially available kit (BIO SOURCE Europe S.A., Nivelles, Belgium). Plasma adrenocorticotropic hormone (ACTH) was determined by 125I-radioimmunoassay with commercially available kit (BRAHMS Aktiengesellschaft, Hennigsdorf, Germany). Serum cortisol was determined by $125 \mathrm{I}$ - radioimmunoassay with commercially available kit (IMMUNOTECH S.A., a Beckman Coulter Company, Prague, Czech Republic). Serum gamma-glutamyl transferase ( $\gamma-G T)$, aspartate transaminase (AST) and alanine transaminase (ALT) were measured photometrically in a Clinical Chemistry Analyzer Z 1145 (Zafiropoulos Diagnostica, Athens, Greece) by the IFCC -UV Kinetic Method with commercially available kits (Zafiropoulos, Athens, Greece).

\subsection{Statistical analysis}

Two-way repeated measures ANOVA was conducted to examine differences in physiological, biochemical and alcohol-related parameters between the control condition and the 8-week ET intervention. If a significant interaction was obtained, pairwise comparisons were performed through simple contrasts and simple main effects analysis using the Bonferroni test method.

One-way repeated measures ANOVA was conducted to examine the effects of the 8-week ET intervention in physiological, biochemical and alcohol-related parameters.

Independent $t$-test was conducted to examine differences between heavy drinkers and matched controls in $\beta$-E levels at the baseline.

Data are presented as mean $\pm \mathrm{SE}$. The level of statistical significance was set at $p<0.05$. The statistical software used for all analyses was SPSS version 18.0 (SPSS Inc., USA).

\section{Results}

\subsection{Control condition vs pre-exercise training intervention}

Statistical analysis did not reveal any significant difference between conditions in baseline values of the parameters examined. Moreover, no significant difference in any parameter before and after the control condition was observed, indicating that during that period no changes in physiological, biochemical or other parameters tested occurred (these results are not presented here).

\subsection{Exercise training intervention}

\subsubsection{Exercise-related parameters}

A significant effect of time for frequency of ET sessions per week was observed $[\mathrm{F}(2,20)=9.121, p=0.005]$. Pairwise comparisons showed significantly increased post-intervention levels compared to pre-intervention $(p<0.05)$. Moreover, a significant effect of time for duration of sessions of ET per week was observed $[\mathrm{F}(2,20)=37.063$, $p<0.001]$. Pairwise comparisons revealed significantly increased mid-intervention levels compared to pre-intervention $(p<0.001)$, and significantly increased post-intervention levels compared to pre-intervention $(p<0.001)$ (Table 1$)$.

\subsubsection{Physiological parameters}

Exercise training resulted in significant changes in some physiological parameters (Table 2). A significant effect of time for weight was observed $[\mathrm{F}(2,20)=5.626, p<0.05]$. Pairwise comparisons revealed a significant decrease in post-intervention levels compared to pre-intervention $(p<0.05)$.

There was a significant effect of time for BMI $[F(2,20)=5.508$, $p<0.05]$. Pairwise comparisons showed that there was a non-significant $(p=0.057)$ decrease in post-intervention levels compared to mid-intervention, and a non-significant $(p=0.058)$ decrease in postintervention levels compared to pre-intervention.

Table 1

Exercise-related parameters of the subjects during exercise training intervention $(\mathrm{M} \pm \mathrm{SE})$.

\begin{tabular}{llll}
\hline Parameter & Pre-intervention & Mid-intervention & Post-intervention \\
\hline $\begin{array}{c}\text { Frequency of ET } \\
\quad \text { (sessions/week) }\end{array}$ & $1.27 \pm 0.54$ & $2.61 \pm 0.31$ & $3.36 \pm 0.24^{\mathrm{a}}$ \\
$\begin{array}{c}\text { Duration of ET (min/ } \\
\text { week) }\end{array}$ & $19.09 \pm 7.80$ & $96.96 \pm 11.48^{\mathrm{a}}$ & $136.64 \pm 16.34^{\mathrm{a}}$ \\
$\begin{array}{c}\text { Mean HR during ET/ } \\
\text { week }\end{array}$ & $133.82 \pm 2.47$ & $134.05 \pm 3.69$ & $137.43 \pm 4.50$ \\
& & &
\end{tabular}

ET: exercise training; HR: heart rate.

${ }^{\text {a }}$ Significant difference from pre-intervention. 
Table 2

Physiological parameters of the subjects throughout exercise training intervention $(\mathrm{M} \pm \mathrm{SE})$

\begin{tabular}{llll}
\hline Parameter & Pre-intervention & Mid-intervention & Post-intervention \\
\hline $\mathrm{VO}_{2 \max }(\mathrm{ml} / \mathrm{kg} / \mathrm{min})$ & $45.76 \pm 1.73$ & $46.27 \pm 1.51$ & $49.17 \pm 2.46$ \\
Weight $(\mathrm{kg})$ & $88.85 \pm 3.05$ & $89.14 \pm 2.91$ & $87.62 \pm 3.04^{\mathrm{a}}$ \\
$\mathrm{BMI}(\mathrm{kg} / \mathrm{m} 2)$ & $28.42 \pm 0.86$ & $28.52 \pm 0.84$ & $28.03 \pm 0.86$ \\
Systolic BP $(\mathrm{mm} \mathrm{Hg})$ & $121.10 \pm 2.47$ & $115.00 \pm 2.69$ & $114.10 \pm 1.78$ \\
Diastolic BP (mm Hg) & $80.70 \pm 2.45$ & $77.00 \pm 2.13$ & $74.10 \pm 1.78$ \\
Waist (cm) & $97.45 \pm 3.61$ & $94.70 \pm 3.13^{\mathrm{a}}$ & $92.70 \pm 3.46^{\mathrm{a}}$ \\
Hip (cm) & $104.58 \pm 1.33$ & $103.89 \pm 0.68$ & $102.28 \pm 0.72$ \\
WHR & $0.88 \pm 0.03$ & $0.86 \pm 0.03$ & $0.86 \pm 0.04$ \\
Flexibility (cm) & $10.22 \pm 2.52$ & $13.11 \pm 2.56^{\mathrm{a}}$ & $16.39 \pm 2.54^{\mathrm{a}}$, b \\
Handgrip (kg) & $46.03 \pm 1.67$ & $45.00 \pm 3.35$ & $47.70 \pm 1.79$ \\
Sit-ups & $27.70 \pm 3.18$ & $35.00 \pm 2.30^{\mathrm{a}}$ & $37.30 \pm 3.41^{\mathrm{a}}$ \\
Push-ups & $15.89 \pm 2.38$ & $17.67 \pm 2.39$ & $19.22 \pm 3.33$ \\
IPAQ (MET-min/week) & $546 \pm 119$ & - & $1663 \pm 271^{\mathrm{a}}$ \\
\hline
\end{tabular}

BMI: Body Mass Index; BP: Blood Pressure; WHR: Waist to Hip Ratio; IPAQ: International Physical Activity Questionnaire.

a Significant difference from pre-intervention.

b Significant difference from mid-intervention.

There was a significant effect of time for systolic BP $[\mathrm{F}(2,18)$ $=4.318, p<0.05]$. Pairwise comparisons revealed a trend for a significant decrease in mid-intervention levels compared to pre-intervention $(p=0.07)$.

A significant effect of time for waist circumference was found [F $(2,20)=11.275, p=0.001]$. Pairwise comparisons revealed a significant decrease $(p<0.05)$ in mid-intervention levels compared to pre-intervention, and a significant decrease $(p<0.01)$ in post-intervention levels compared to pre-intervention.

A significant effect of time for flexibility was found $[\mathrm{F}(2,16)$ $=14.483, p<0.001]$. Pairwise comparisons showed that there were significantly increased $(p<0.05)$ mid-intervention levels compared to pre-intervention, significantly increased $(p<0.05)$ post-intervention levels compared to mid-intervention, and significantly increased ( $p=0.01$ ) post-intervention levels compared to pre-intervention.

A significant effect of time for sit-ups was observed $[\mathrm{F}(2,18)$ $=8.852, p<0.005]$. Pairwise comparisons revealed a significant increase $(p<0.05)$ in mid-intervention levels compared to pre-intervention, and a significant increase $(p<0.05)$ in post-intervention levels compared to pre-intervention.

Finally, paired $t$-test revealed significantly increased $(p=0.001)$ post-intervention IPAQ values compared to pre-intervention (pre: $546 \pm 119$ vs. post: $1663 \pm 271$ ). Detailed results are presented in Table 2.

\subsubsection{Biochemical parameters}

A significant effect of time for $\gamma$-GT was observed $[F(2,20)=5.880$, $p<0.05]$. Pairwise comparisons revealed a significant decrease $(p<0.01)$ in post-intervention levels compared to pre-intervention. No significant difference $(p>0.05)$ was found for any of the other biochemical parameters assessed. Detailed results are presented in Table 3.

\subsubsection{Alcohol-related and other outcomes}

There was a positive effect of the 8-week ET intervention on subjects' behavior towards alcohol, as indicated by the alcohol use questionnaire scores. In the majority of the questions regarding alcohol intake, subjects reported significantly lower intake; however, the intention to decrease or stop drinking was not affected (Table 4).

Pearson correlation revealed that there was a negative correlation $(r=-0.441 ; p=0.01$ ) between the exercise frequency per week and the AU consumed per week. There was also a non-significant negative correlation $(r=-0.341 ; p=0.52)$ between the exercise frequency per week and the AU usually consumed per week. No significant correlation between exercise duration per week and alcohol-related parameters was observed.

Concerning the alcohol units consumed weekly, a significant decrease $(p<0.05)$ in post-intervention levels compared to pre-intervention was observed. Concerning the desire for alcohol over the last week, a trend for a significant decrease $(p=0.06)$ in post-intervention levels compared to mid-intervention was observed. Moreover, a significant decrease $(p<0.005)$ in post-intervention AUDIT values compared to pre-intervention was found (pre: $17.5 \pm 1.6$ vs. post: $12.8 \pm 2.1$ ) (Table 5).

\section{Discussion}

To our knowledge this is the first study that investigated the effects of ET on physiological mechanisms related to alcohol use disorders in humans. Results indicate that even though positive changes are seen in physiological parameters and alcohol intake habits with ET this is not related with hormonal modifications.

The 8-week ET intervention resulted in improvement in several indices of fitness in heavy drinkers. Similar gains have been reported in previous studies [29-35], whereas one study [36] did not find significant change.

More specifically, a positive effect of the 8-week ET intervention on body composition was indicated by changes in body weight and waist circumference, as well as a non-significant change in BMI. Subjects had greater waist circumference compared to controls at the baseline, while the ET intervention resulted in increased physical activity levels along with decreased weight and waist circumference, and non-significantly decreased BMI. It is well established that ET contributes to weight control and leads to beneficial metabolic changes in both healthy and non-healthy populations. However, concerning individuals with AUDs, we found in the literature only one study that reported reduced body fat percentage after ET intervention [35].

Table 3

Biochemical parameters of the subjects throughout exercise training intervention $(M \pm S E)$.

\begin{tabular}{|c|c|c|c|c|}
\hline Index & Pre-intervention & Mid-intervention & Post-intervention & Normal range \\
\hline$\gamma$-GT (U/L) & $59.75 \pm 10.35$ & $54.70 \pm 10.95$ & $49.00 \pm 9.59^{\mathrm{a}}$ & $11-61$ (men) \\
\hline AST (U/L) & $29.25 \pm 4.20$ & $28.49 \pm 2.60$ & $30.19 \pm 1.96$ & $\leq 37$ (men) \\
\hline ALT (U/L) & $29.27 \pm 3.98$ & $29.42 \pm 3.58$ & $26.86 \pm 4.23$ & $\leq 42$ (men) \\
\hline ACTH (pg/ml) & $30.91 \pm 4.02$ & $36.09 \pm 4.93$ & $34.46 \pm 4.26$ & 10-60 (8-10 a.m.) \\
\hline $\mathrm{E}(\mathrm{pg} / \mathrm{ml})$ & $43.64 \pm 3.58$ & $42.46 \pm 4.54$ & $40.82 \pm 3.52$ & $<100$ \\
\hline $\mathrm{NE}(\mathrm{pg} / \mathrm{ml})$ & $275.00 \pm 35.01$ & $254.36 \pm 29.41$ & $242.00 \pm 28.91$ & $<600$ \\
\hline Dopamine $(\mathrm{pg} / \mathrm{ml})$ & $39.46 \pm 2.64$ & $41.18 \pm 3.36$ & $47.82 \pm 6.22$ & $<100$ \\
\hline Cortisol (nM) & $151.10 \pm 27.71$ & $158.00 \pm 18.11$ & $167.30 \pm 17.69$ & 260-720 (morning) \\
\hline$\beta-\mathrm{E}(\mathrm{pg} / \mathrm{ml})$ & $3.31 \pm 0.40$ & $4.28 \pm 0.71$ & $3.48 \pm 0.64$ & $3.84 \pm 0.92$ (matched controls) \\
\hline Cortisol/ACTH & $4.92 \pm 0.92$ & $4.95 \pm 0.62$ & $5.35 \pm 0.64$ & \\
\hline
\end{tabular}

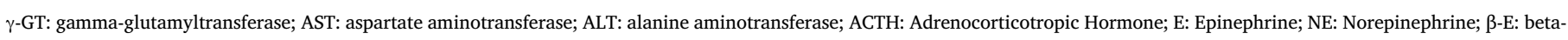
endorphin.

a Significant difference from pre-intervention. 
Table 4

Alcohol use questionnaire scores of the subjects throughout exercise training intervention ( \pm SE).

\begin{tabular}{|c|c|c|c|}
\hline Question & Pre-intervention & Mid-intervention & Post-intervention \\
\hline How many AU do you drink per day? & $3.05 \pm 0.61$ & $2.96 \pm 0.63$ & $1.96 \pm 0.43^{\mathrm{a}}$ \\
\hline How many AU did you drink last night? & $2.55 \pm 0.46$ & $1.55 \pm 0.47$ & $2.46 \pm 0.71$ \\
\hline How many times did you consume alcohol over the last month? & $6.82 \pm 0.33$ & $5.82 \pm 0.46^{\mathrm{a}}$ & $5.27 \pm 0.43^{\mathrm{a}}$ \\
\hline How many AU did you use to drink per occasion over the last month? & $5.58 \pm 0.51$ & $4.27 \pm 0.43^{\mathrm{a}}$ & $3.68 \pm 0.49^{\mathrm{a}}$ \\
\hline How many days do you usually drink alcohol? & $5.14 \pm 0.46$ & $4.55 \pm 0.53$ & $3.86 \pm 0.68^{\mathrm{a}}$ \\
\hline How many AU do you usually drink per week? & $19.00 \pm 3.20$ & $15.86 \pm 3.04$ & $11.64 \pm 3.03^{\mathrm{a}}$ \\
\hline Would you like to stop drinking alcohol? $(1-10)$ & $3.91 \pm 0.98$ & $4.46 \pm 1.00$ & $5.18 \pm 0.91$ \\
\hline Would you like to cut down on alcohol? (1-10) & $5.73 \pm 0.91$ & $5.64 \pm 0.87$ & $6.73 \pm 0.81$ \\
\hline Time until first drink after an exercise session (min) & $592 \pm 88.4$ & $1170 \pm 387.6$ & $1370 \pm 311.6^{\mathrm{a}}$ \\
\hline
\end{tabular}

AU: Alcohol Units (1 $\mathrm{AU}=14 \mathrm{~g}$ of pure alcohol). 1: Not much; 10: Very much.

${ }^{\text {a }}$ Significant difference from pre-intervention.

Moreover, systolic BP decreased by $7 \mathrm{~mm} \mathrm{Hg}$ (approximately 6\%) and these changes almost reached significance. Only two previous studies have investigated possible changes in BP of individuals with AUDs by ET and provided inconsistent results; one study reported decreased systolic BP [34], whereas the other one did not report any significant changes [37]. Possible explanations for this result is that BP of the subjects that participated in the present study was within the normal limits at the baseline and only a slight decrease could be obtained after intervention or significant results could be obtained if the time period of intervention was longer. However, it has been demonstrated that even a $2 \mathrm{~mm} \mathrm{Hg}$ reduction in SPB may result in reduced risk of cardiovascular morbidity associated with elevated blood pressure (e.g. stroke, ischemic heart disease) [38].

There are mixed results concerning the effect of the 8-week ET intervention on parameters of physical conditioning. After ET intervention, physical activity level, flexibility and sit-ups values significantly increased, whereas $\mathrm{VO}_{2 \max }$, handgrip and push-ups values did not change. Literature also provides inconsistent evidence regarding the effect of ET interventions on physical functioning in individuals with AUDs. A previous study by Palmer et al. [36] reported no changes in the values of $\mathrm{VO}_{2 \max }$, while most studies have reported a significant positive effect of $\mathrm{ET}$ on $\mathrm{VO}_{2 \max }[30,33,39,40]$. Moreover, two previous studies have reported significantly increased muscular strength of subjects in intervention groups [33,39]. It is likely that a combination of aerobic with resistance exercise could lead to positive effects in extended parameters of physical conditioning.

Physical activity levels at the end of the 8-week ET intervention were 3 times higher from the baseline levels. This is in agreement with previous studies that have reported increased levels of physical activity achieved after ET intervention [33,39,40]. Engagement of individuals in ET programmes has been shown to increase their self-confidence, self-esteem and self-efficacy levels [41,42]. Motivation of the subjects to increase the frequency and duration of exercise sessions could explain our results. All these favorable physiological outcomes in combination with decreased alcohol consumption after ET intervention indicate that exercise is beneficial for heavy drinkers and highlight the importance of including ET intervention in the treatment of AUDs.

Central $\beta$-E deficiency has been previously shown to occur both in human alcoholics and in animal models, and may contribute to increased alcohol urge and relapse rates [4]. However, various studies on the effect of chronic exposure to alcohol on the pituitary and hypothalamic $\beta$-E systems have provided inconsistent results that are attributed to methodological problems [4].

A previous study from our laboratory [9] has reported that alcoholics had lower plasma $\beta$-E levels compared to healthy counterparts. The baseline $\beta$-E levels at rest of heavy drinkers that participated in the present study were not significantly different from those in the control group, indicating that there may be a central $\beta$-E deficiency in alcoholics but not in heavy drinkers. The different levels of baseline $\beta$-E levels between alcoholics and heavy drinkers could be explained by the different level of exposure to alcohol. Moreover, a 29.2\% non-significant increase in $\beta$-E levels at the 4th week of ET intervention was detected; however, $\beta$-E levels decreased again reaching the baseline levels at the 8th week of ET intervention. From these results one can assume that chronic exercise of moderate intensity may only lead to medium-term increases in $\beta$-E levels of heavy drinkers. Furthermore, heavy drinkers may not experience a central $\beta$-E deficiency and, therefore, ET intervention may not have a significant effect on the opioid system.

In addition, the effects of alcohol on the body mainly depend on the quantity consumed, the pattern of drinking and the duration of exposure. Gender differences in $\beta$-E levels at rest may also exist; however, in the present study only male heavy drinkers were included. Previous studies have demonstrated that female heavy drinkers have lower plasma $\beta$-E and ACTH levels than male counterparts [43]. In addition to that, genetic factor may also affect $\beta$-E levels at rest since it was shown that individuals with a family history of alcoholism exhibit lower plasma $\beta$-E [44] and ACTH [45] levels than those with no family history of alcoholism. Since heavy drinkers that participated in the present study did not report a family history of alcoholism, this could also be a reason for the lack of differences in their baseline levels of $\beta$-E and ACTH compared to the control group.

Increased release of cortisol from the adrenal cortex can be induced by various stressful conditions, such as excessive alcohol drinking. Alcoholics may exhibit increased levels of cortisol which exert a glucocorticoid negative feedback both at the hypothalamus and pituitary,

Table 5

Alcohol-related parameters of the subjects throughout exercise training intervention $(\mathrm{M} \pm \mathrm{SE})$.

\begin{tabular}{|c|c|c|c|}
\hline Parameter & Pre-intervention & Mid-intervention & Post-intervention \\
\hline AU consumed over the last week & $18.64 \pm 3.69$ & $16.73 \pm 4.61$ & $12.55 \pm 2.65^{\mathrm{a}}$ \\
\hline Desire for alcohol over the last week (0: No desire - 10: Strong desire) & $7.27 \pm 0.45$ & $7.27 \pm 0.47$ & $6.46 \pm 0.55^{\mathrm{a}}$ \\
\hline Goal setting for alcohol moderation (0: No goal - 10: Big goal) & $3.18 \pm 0.46$ & $2.55 \pm 0.28$ & $2.73 \pm 0.33$ \\
\hline How sure are you that you can achieve your goal? (0: Not sure - 10 : Totally sure) & $6.73 \pm 0.63$ & $7.00 \pm 0.74$ & $7.64 \pm 0.69$ \\
\hline AUDIT score $(0-20)$ & $17.5 \pm 1.6$ & - & $12.8 \pm 2.1^{\mathrm{a}}$ \\
\hline
\end{tabular}

AU: Alcohol Units (1 AU = $14 \mathrm{~g}$ of pure alcohol); AUDIT: Alcohol Use Identification Test.

a Significant difference from pre-intervention. 
resulting in decreased ACTH secretion. The results of the present study showed that ACTH and cortisol levels in heavy drinkers were within normal limits and did not change throughout ET intervention. This could also be explained by the lower exposure to alcohol of heavy drinkers compared to alcoholics.

Changes in the levels of hormones of the HPA axis can reflect changes in the function of central nervous system (CNS). The 8-week ET intervention had no effect on any of those indicators of HPA axis activity in heavy drinkers. The sympathetic nervous system plays an important role in mediating the response to exercise in healthy individuals [46], and abnormal response of this system both at rest and during physical exercise indicates abnormal cardiac response, something that was not evident in heavy drinkers. More research on the effect of ET on HPA-axis activation in individuals with AUDs is warranted.

Heavy drinkers often suffer from an AUD; however, many of them abuse alcohol over a long period of time without becoming addicted to alcohol [47]. The pathophysiology of alcohol misuse is very complicated and the exact physiological mechanism by which heavy drinkers eventually become addicted to alcohol is not known yet. There is no known specific neurotransmitter binding site in the brain for alcohol, unlike most illicit drugs of abuse. For that reason, research on alcohol dependence is mainly focused on the effects of alcohol in pathways of neuronal communication that integrate the activities of multiple brain regions.

The mesolimbic dopamine system (MDS), the most important reward pathway in the brain that carries dopamine from one area of the brain to another, is thought to be involved in the development of substance dependence, including alcohol. Alcohol misuse may gradually impair MDS function, which in turn results in rewarding and addictive effects [48]. Alcohol promotes dopamine release in the nucleus accumbens, a brain region and part of the MDS with many neurons that contain dopamine receptors; however, chronic excessive exposure to alcohol may decrease the number of dopamine receptors in alcoholic patients [49]. Exercise may also activate the MDS. It has been proposed that since dopaminergic reinforcement mechanisms in the neural system are activated by both alcohol and exercise, alcohol consumption and exercise may produce similar pleasurable effects [50,51], rending exercise a useful tool for the prevention and treatment of AUDs. Although previous ET interventions in animal models have reported sustained increases in the number of dopamine receptors in the striatum $[52,53]$, no change in plasma dopamine levels after 8 weeks of ET intervention in heavy drinkers was found in the present study. This finding could be explained by the fact that plasma dopamine levels may not reflect the quantity of dopamine receptors in the brain or the lower exposure to alcohol of heavy drinkers compared to alcoholics.

Chronic excessive exposure to alcohol is a causative factor of liver inflammation. $\gamma$-GT levels is an indicator of liver inflammation that can reflect changes in alcohol use pattern. The subjects had $\gamma$-GT levels at the higher end of the normal range at the baseline, while the 8-week ET intervention resulted in decreased $\gamma$-GT levels along with reduced alcohol intake. Therefore, it is likely that the main factor that influenced the decreased $\gamma$-GT levels in heavy drinkers was the reduced alcohol intake. Moreover, liver transaminases (AST and ALT), especially ALT, are also indicators of liver inflammation [54]. AST and ALT levels were within the normal limits throughout the ET intervention. It has been suggested that central adiposity, independently from BMI, is associated with greater levels of liver enzymes, which may be the result of unrecognized fatty liver [55]. Obese individuals may be at greater risk of developing fatty liver than heavy drinkers; however, individuals with both obesity and heavy alcohol use may be at even greater risk of developing this condition [56]. The subjects of the present study were considered overweight according to BMI (BMI $=28.42 \pm 0.86 \mathrm{~kg} / \mathrm{m}^{2}$ ) but their WHR was within the normal limits. Thus it could be hypothesized that changes in liver enzyme levels could reflect changes in the amounts of alcohol consumed.

The intervention was an 8-week supervised aerobic ET with exercise sessions of increasing frequency and duration, where a minimum of two 30-min supervised exercise sessions per week was set. Previous intervention studies involved three to sixteen weeks of ET of mostly aerobic exercise $(55 \%-85 \%$ of HRmax), one to five times per week $[29-33,35,36,40,57]$. The subjects that participated in the present study were motivated to increase the frequency and duration of exercise sessions as part of long-term lifestyle modifications that include increased physical activity and reduced alcohol consumption. For that reason, the subjects were asked to set a goal for exercise duration and alcohol consumption before each session. Therefore, individuals were free to decide which exercise duration and frequency was the more suitable and pleasant for them, in order to increase the possibility of achieving these long-term lifestyle modifications. Heart rate was monitored during supervised exercise sessions and it was estimated that all subjects were exercising at moderate intensity (50-60\% of Heart Rate Reserve) throughout the ET intervention.

AUDIT scores decreased significantly following the ET intervention. Alcohol-related parameters are very important in the treatment of AUDs because they provide useful information on the efficacy of interventions aimed at alcohol abuse cessation. Desire for alcohol, which plays an important role in the treatment of AUDs, was significantly decreased after ET intervention. Interestingly, heavy drinkers reported that their will to cut down on alcohol consumption was low throughout the ET intervention, whereas alcohol consumption was significantly decreased at the end of ET intervention. It would be expected that a decrease in alcohol consumption would be accompanied by increased will of heavy drinkers to cut down on alcohol; however, no such result was observed. It is possible that other physiological and/or psychological factors may have contributed to these findings and certainly this needs to be examined further. Even though there were no significant differences under basal conditions in hormonal levels there may be changes in the responses of some of these hormones in response to stressful situations physical (such as exercise) or psychological. Thus, the increased ET participation of the subjects may increase their ability to cope with stressful situations which could be partially responsible for their decreased alcohol consumption. This is an aspect that needs further investigation. Previous studies have also examined alcohol-related parameters after exercise such as alcohol urge, abstinence rates and drinking behavior through questionnaires and/or via biochemical parameters, with positive outcomes observed [30,34,35,40,58].

Subjects were recording their daily alcohol consumption throughout the study. During the control condition, no significant change in alcohol consumption was found; however, gradually decreased alcohol consumption was reported in the ET intervention. Therefore, it is likely that subjects' behavior towards alcohol was not affected by the fact that they had to record their alcohol consumption. Moreover, subjects were aware of their drinking problem and it could be assumed that they would make an effort to cut down on alcohol consumption anyway. Nevertheless, their intention to quit or cut down on alcohol was reported to be medium and did not change after ET intervention. All these results indicate that the decreased alcohol consumption reported was influenced by the ET intervention.

Since alcohol consumption was self-reported, the results could be inaccurate. For that reason, changes in $\gamma$-GT levels, which reflect changes in alcohol consumption, were examined. It was observed that changes in $\gamma$-GT levels were similar to changes in alcohol consumption; alcohol consumption and $\gamma$-GT levels decreased after ET intervention. Therefore, it is likely that self-reported alcohol consumption was in agreement with the actual amounts of alcohol consumed.

During the ET intervention, $15.4 \%$ of the subjects (2 out of 13) dropped out. This drop-out rate is lower in comparison to findings from previous studies; a 16-week ET intervention reported 43.5\% drop-out rate [31] and a 4-week ET intervention reported $26 \%$ drop-out rate [33].

Although the results indicate that the 8-week ET intervention was effective in decreasing alcohol consumption while increasing physical 
activity, there were some limitations. One of these limitations is that there was a small sample; randomized controlled studies with a larger sample size could provide more reliable results. Moreover, only male heavy drinkers were included in the study and, therefore, findings of the ET intervention cannot be generalized for female heavy drinkers. It is known that women are more vulnerable to the effects of alcohol consumption than men due to physiological differences. In a recent study by Gallego et al. [59]., it was reported that there was a significant decrease in ethanol consumption and preference in female but not in male mice following voluntary exercise, indicating that there may be gender differences in the efficacy of voluntary exercise and its effects on alcohol-related behaviors. Moreover, examination of biochemical indices obtained from other tissues than blood could offer a better understanding of the physiological mechanisms involved in individuals with AUDs who participate in ET interventions. Indirect measurements in the plasma may not correlate with the levels of $\beta$-E and other endogenous opioids in the brain; however, direct measurement of these compounds in the living human brain is not possible. Finally, in this study there was a direct comparison of the experimental group with a control group only for the baseline measurements. A better understanding of the physiology and behavior would be gained if an "alcoholmatched" group of individuals was included, i.e. individuals who drink less over an 8-week period, but who do not exercise, and compare them to the exercising group. This consists of a limitation of the study but it is a topic of further investigation.

\section{Conclusions}

It is concluded that an 8-week supervised aerobic ET intervention may be beneficial for individuals with AUDs in terms of long-term lifestyle modifications; however, this seems not to be related with changes in hormonal responses associated with the HPA-axis.

Although only a small number of studies on the effects of exercise on alcohol use and related parameters in individuals with AUDs exist, the limited available data indicate that this is a promising research topic. ET interventions that include motivation goals may be more effective in achieving and maintaining a healthier lifestyle in individuals with AUDs than only exercise or psychological support. Future large-scale studies that would also examine gender differences should be conducted.

\section{Funding}

This study was co-financed by the European Union [European Social Fund (ESF)] (MIS 375607) and Greek national funds through the Operational Program 'Education and Lifelong Learning' of the National Strategic Reference Framework-Research Funding Program: Thales (730). Investing in knowledge society through the ESF.

\section{Disclosure statement}

The authors declare no conflicts of interest.

\section{Author contributions}

A.Z.J. and Y.T. conceived and designed the experiments; K.G. and E.M. performed the experiments; K.G. P.G. and A.Z. analyzed the data; K.G., I.G.F, G.M., Y.K. and A.Z.J. wrote the paper.

\section{References}

[1] S.E. Brien, P.E. Ronksley, B.J. Turner, K.J. Mukamal, W.A. Ghali, Effect of alcohol consumption on biological markers associated with risk of coronary heart disease: systematic review and meta-analysis of interventional studies, Br. Med. J. 342 (2011) d636.

[2] W. Caan, J. de Belleroche, Drink, Drugs and Dependence: From Science to Clinical Practice, 1st ed., Routledge, New York, 2002, pp. 19-20.
[3] World Health Organization, Global Status Report on Alcohol and Health, WHO Press, Geneva, 2011.

[4] C. Gianoulakis, Endogenous opioids and addiction to alcohol and other drugs of abuse, Curr. Top. Med. Chem. 4 (1) (2004) 39-50.

[5] F. Noble, M. Lenoir, N. Marie, The opioid receptors as targets for drug abuse medication, Br. J. Pharmacol. 172 (16) (2015) 3964-3979.

[6] L.D. Keith, J.C. Crabbe, L.M. Robertson, J.W. Kendall, Ethanol stimulated endorphin and corticotrophin secretion in vitro, Brain Res. 367 (1986) 222-229.

[7] A.B. Thiagarajan, I.N. Mefford, R.L. Eskay, Single-dose ethanol administration activates the hypothalamic-pituitary-adrenal axis: exploration of the mechanism of action, Neuroendocrinology 50 (4) (1989) 427-432.

[8] C. Gianoulakis, Characterization of the effects of acute ethanol administration on the release of beta-endorphin peptides by the rat hypothalamus, Eur. J. Pharmacol. 180 (1) (1990) 21-29.

[9] A.Z. Jamurtas, N. Zourbanos, K. Georgakouli, P. Georgoulias, E. Manthou, I.G. Fatouros, et al., Beta endorphin and alcohol urge responses in alcoholic patients following an acute bout of exercise, J. Addict. Res. Ther. 5 (2014) 194.

[10] A.L. Errico, A.C. King, W.R. Lovallo, O.A. Parsons, Cortisol dysregulation and cognitive impairment in abstinent male alcoholics, Alcohol. Clin. Exp. Res. 26 (8) (2002) 1198-1204.

[11] E. Zschucke, A. Heinz, A. Ströhle, Exercise and physical activity in the therapy of substance use disorders, ScientificWorldJournal 2012 (2012) 901741.

[12] M.E. Donaghy, G. Ralston, N. Mutrie, Exercise as a therapeutic adjunct for problem drinkers, J. Sports Sci. 9 (1991) 440.

[13] M.E. Donaghy, N. Mutrie, Is exercise beneficial in the treatment and rehabilitation of the problem drinker? A critical review, Phys. Ther. Rev. 4 (1999) 153-166.

[14] J.P. Read, R.A. Brown, The role of physical exercise in alcoholism treatment and recovery, Prof. Psychol. Res. Pr. 34 (1) (2003) 49-56.

[15] C.P. O'Brien, D.R. Gastfriend, R.F. Forman, E. Schweizer, H.M. Pettinati, Long-term opioid blockade and hedonic response: preliminary data from two open-label extension studies with extended-release naltrexone, Am. J. Addict. 20 (2011) $106-112$.

[16] L.L. Craft, F.M. Perna, The benefits of exercise for the clinically depressed, Prim. Care Companion J. Clin. Psychiatry 6 (3) (2004) 104-111.

[17] A. Broocks, B. Bandelow, G. Pekrun, A. George, T. Meyer, U. Bartmann, et al., Comparison of aerobic exercise, clomipramine, and placebo in the treatment if panic disorder, Am. J. Psychiatry 155 (1998) 603-609.

[18] G. Papathanasiou, G. Georgoudis, M. Papandreou, P. Spyropoulos, D. Georgakopoulos, V. Kalfakakou, et al., Reliability measures of the short International Physical Activity Questionnaire (IPAQ) in Greek young adults, Hellenic J. Cardiol. 50 (4) (2009) 283-294.

[19] National Institute on Alcohol Abuse and Alcoholism, Drinking levels defined. http://www.niaaa.nih.gov/alcohol-health/overview-alcohol-consumption/ moderate-binge-drinking, (2016) (accessed 10.10.2016).

[20] G. Moussas, G. Dadouti, A. Douzenis, E. Poulis, A. Tzelembis, D. Bratis, et al., The alcohol use disorders identification test (AUDIT): reliability and validity of the Greek version, Ann. General Psychiatry 8 (2009) 11.

[21] T.F. Babor, J.C. Higgins-Biddle, J.B. Saunders, M.G. Monteiro, World Health Organization, WHO Department of Mental Health and Substance Dependence. The Alcohol Use Disorders Identification Test. Guidelines for Use in Primare Care, 2nd ed., WHO Press, Geneva, 2001.

[22] T.F. Babor, R. Campbell, R. Room, J.B. Saunders, World Health Organization, Lexicon of Alcohol and Drug Terms, WHO Press, Geneva, 1994.

[23] World Health Organization, The ICD-10 Classification of Mental and Behavioural Disorders: Diagnostic Criteria for Research, WHO Press, Geneva, 1993.

[24] United Nations Educational, Scientific and Cultural Organization, International Standard Classification of Education, UNESCO Institute for Statistics, Montreal, 2012.

[25] US National Institutes of Health, ClinicalTrials.gov., http://www.clinicaltrials.gov; 2016 (accessed 12.10.2016)

[26] C.B. Ebbeling, A. Ward, E.M. Puleo, J. Widrick, J.M. Rippe, Development of a single-stage submaximal treadmill walking test, Med. Sci. Sports Exerc. 23 (8) (1991) 966-973.

[27] K.F. Wells, E.K. Dillon, The sit and reach. A test of back and leg flexibility, Res. Quart. 23 (1952) 115-118.

[28] K. Georgakouli, E. Manthou, I.G. Fatouros, C.K. Deli, D.A. Spandidos, A.M. Tsatsakis, et al., Effects of acute exercise on liver function and blood redox status in heavy drinkers, Exp. Ther. Med. 10 (6) (2015) 2015-2022.

[29] V. Gary, D. Guthrie, The effect of jogging on physical fitness on self-concept on hospitalized alcoholics, Q. J. Stud. Alcohol 33 (4) (1972) 1073-1078.

[30] D. Sinyor, T. Brown, L. Rostant, P. Seraganian, The role of physical fitness program in the treatment of alcoholism, J. Stud. Alcohol 43 (1982) 380-386.

[31] A. Weber, Running as treatment for hospitalized alcoholics: an experimental approach, Suchtgefahren 30 (3) (1984) 160-167 (German).

[32] T.J. Murphy, R.R. Pagano, G.A. Marlatt, Lifestyle modification with heavy alcohol drinkers: effects of aerobic exercise and meditation, Addict. Behav. 11 (2) (1986) $175-186$.

[33] M.E. Donaghy, The Investigation of Exercise as an Adjunct to the Treatment and Rehabilitation of the Problem Drinker, dissertation University of Glasgow, UK, 1997.

[34] R. Ermalinski, P.G. Hanson, J.B. Lubin, J.I. Thornby, P.A. Nahormek, Impact of a body-mind treatment component on alcoholic patients, J. Psychosoc. Nurs. 35 (7) (1997) 39-45.

[35] R.A. Brown, A.M. Abrantes, J.P. Read, B.H. Marcus, J. Jakicic, D.R. Strong, et al., Aerobic exercise for alcohol recovery: rationale, program description, and preliminary findings, Behav. Modif. 33 (2) (2009) 220-249. 
[36] J. Palmer, N. Vacc, J. Epstein, Adult impatient alcoholics: physical exercise as a treatment intervention, J. Stud. Alcohol 49 (1988) 418-421.

[37] M. Lehofer, M. Lux, C. Posch, J. Berthold, H. Wieser, G. Hirn, et al., Lauftherapie im Entzug bei chronischem Alkoholismus, Wiener Zeitschrift für Suchtforschung 1/2 (18) (1995) 55-64 ((S). German).

[38] Prospective Studies Collaboration, Age-specific relevance of usual blood pressure to vascular mortality: a meta-analysis of individual data for one million adults in 61 prospective studies, Lancet 360 (9349) (2002) 1903-1913.

[39] E.M. Capodaglio, G. Vittadini, D. Bossi, S. Sverzellati, M. Facioli, C. Montomoli, et al., A functional assessment methodology for alcohol dependent patients undergoing rehabilitative treatments, Disabil. Rehabil. 25 (21) (2003) 1224-1230.

[40] R.A. Brown, A.M. Abrantes, H. Minami, J.P. Read, B.H. Marcus, J.M. Jakicic, et al., A preliminary, randomized trial of aerobic exercise for alcohol dependence, J. Subst. Abus. Treat. 47 (1) (2004) 1-9.

[41] S.A. Paluska, T.L. Schwenk, Physical activity and mental health: current concepts, Sports Med. 29 (3) (2000) 167-180.

[42] D.M. Landers, S.M. Arent, Physical activity and mental health, in: H.A. Hausenblas, C.M. Janelle (Eds.), Handbook of Research in Sport Psychology, 2nd ed., John Wiley and Sons, New York, 2001, pp. 740-765.

[43] C. Gianoulakis, X. Dai, T. Brown, Effect of chronic alcohol consumption on the activity of the hypothalamic-pituitary-adrenal axis and pituitary $\beta$-endorphin as a function of alcohol intake, age, and gender, Alcohol. Clin. Exp. Res. 27 (3) (2003) $410-423$.

[44] C. Gianoulakis, Influence of the endogenous opioid system on high alcohol consumption and genetic predisposition to alcoholism, J. Psychiatry Neurosci. 26 (4) (2001) 304-318

[45] X. Dai, J. Thavundayil, C. Gianoulakis, Response of the HPA-axis to stress and al cohol as a function of chronic alcohol abuse and family history of alcoholism, Alcohol Clin. Exp. Res. (Suppl. 26) (2002) 146A.

[46] U.S. van Euler, S. Hellner, Excretion of noradrenaline and adrenaline in muscula work, Acta Physiol. Scand. Suppl. 26 (1952) 183-191.

[47] G.E. Vaillant, A 60-year follow-up of alcoholic men, Addiction 98 (8) (2003)
1043-1051.

[48] P.W. Kalivas, N.D. Volkow, The neural basis of addiction: a pathology of motivation and choice, Am. J. Psychiatry 162 (8) (2005) 1403-1413.

[49] N.D. Volkow, J.S. Fowler, G.J. Wang, R. Baler, F. Telang, Imaging dopamine's role in drug abuse and addiction, Neuropharmacology 56 (Suppl. 1) (2009) 3-8.

[50] T.L. Cronan, E.T. Howley, The effect of training on epinephrine and norepinephrine excretion, Med. Sci. Sports 5 (1974) 122-125.

[51] P. Thoren, J.S. Floras, P. Hoffmann, D.R. Seals, Endorphins and exercise: physiological mechanisms and clinical implications, Med. Sci. Sports Exerc. 22 (1990) 417-428.

[52] P.E. Gilliam, W.W. Spirduso, T.P. Martin, T.J. Walters, R.E. Wilcox, R.P. Farrar, The effects of exercise training on $3 \mathrm{H}$-spiperone binding in rat striatum, Pharmacol. Biochem. Behav. 20 (1984) 863-867.

[53] P.G. MacRae, W.W. Spirduso, G.D. Cartee, R.P. Farrar, R.E. Wilcox, Endurance training effects on striatal D2 dopamine receptor binding and striatal dopamine metabolite levels, Neurosci. Lett. 79 (1-2) (1987) 138-144.

[54] G. Banfi, A. Colombini, G. Lombardi, A. Lubkowska, Chapter 1 - Metabolic markers in sports medicine, Adv. Clin. Chem. 56 (2012) 1-54.

[55] S. Stranges, J.M. Dorn, P. Muti, J.L. Freudenheim, E. Farinaro, M. Russell, et al., Body fat distribution, relative weight, and liver enzyme levels: a population-based study, Hepatology 39 (3) (2004) 754-763.

[56] S. Bellentani, G. Saccoccio, F. Masutti, L.S. Croce, G. Brandi, F. Sasso, et al, Prevalence of and risk factors for hepatic steatosis in Northern Italy, Ann. Intern. Med. 132 (2) (2000) 112-117.

[57] P.L. McKelvy, C.A. Stein, A.B. Bertini, Heart-rate response to a conditioning program for young, alcoholic men, Phys. Ther. 60 (2) (1980) 184-187.

[58] M. Ussher, A.K. Sampuran, R. Doshi, R. West, D.C. Drummond, Acute effect of a brief bout of exercise on alcohol urges, Addiction 99 (12) (2004) 1542-1547.

[59] X. Gallego, R.J. Cox, E. Funk, R.A. Foster, M.A. Ehringer, Voluntary exercise decreases ethanol preference and consumption in C57BL/6 adolescent mice: sex differences and hippocampal BDNF expression, Physiol. Behav. 138 (2015) 28-36. 